\title{
Washing Durability of PDMS-Conductive Fabric Composite: Realizing Washable UHF RFID Tags
}

\author{
Roy B. V. B. Simorangkir, Member, IEEE, Duc Le, Toni Björninen, Senior Member, IEEE, Abu Sadat Md. \\ Sayem, Student Member, IEEE, Maxim Zhadobov, Senior Member, IEEE, Ronan Sauleau, Fellow, IEEE
}

\begin{abstract}
In this paper, we present experimental investigations on washing durability of polydimethylsiloxane (PDMS)conductive fabric composite, to validate its applicability for realization of flexible wearable antennas that can withstand multiple washing cycles. For this purpose, we designed an ultrahigh frequency (UHF) radio frequency identification (RFID) passive tag antenna and fabricated several prototypes using such materials combination. Understanding the challenge of having a robust integration of lumped electronic component (e.g., RFID IC) on a flexible antenna, a new way to improve the interconnection has also been investigated. The tag prototypes were subjected to recurrent machine-washing tests, and after each washing cycle, their performance was analyzed mainly in terms of read range. The results reveal that, with a proper treatment on the antenna-IC fixture interconnection, the tag antennas developed with PDMS-conductive fabric composite can maintain their performance very well, showing a minimum degradation in read range after 15 cycles of washing.
\end{abstract}

Index Terms-Conductive fabric, flexible antennas, polydimethylsiloxane (PDMS), radio frequency identification (RFID), recurrent washings, tag antennas, textile antennas.

\section{INTRODUCTION}

$\mathbf{T}$ HE ubiquity of wireless wearable technologies over the last decade has created an ever-growing research interest in the utilization of textiles for wearable antenna realization [1]-[3]. Current research efforts in this area towards the realization of truly wearable wireless platforms include achieving the antenna durability against recurrent machine washings. As part of a daily apparel, a textile-based antenna is expected to be able to withstand frequent washings, which is such a highly challenging task. Exposure to water and other chemicals as well as extreme mechanical stress of the turbulence inside the washing machine often lead to the deterioration of the antenna performance or even threaten its longevity [4]-[6].

Some efforts have been reported to achieve the antenna robustness against frequent washings [4], [5], [7]-[11]. The general solution applied was by covering the whole antenna or only certain part of it (e.g., the antenna-electronic component interconnection in some antenna cases) with various coating materials. As compared to the case where no coating was

R. B. V. B. Simorangkir, M. Zhadobov, R. Sauleau are with University of Rennes 1, CNRS, Institut d'Electronique et de Télécommunications of Rennes (IETR)-UMR 6164, F-35000 Rennes, France (e-mail: roy.simorangkir@ieee.org).

D. Le and T. Björninen and are with Faculty of Medicine and Health Technology, Tampere University, Tampere 33720, Finland (e-mail: vietduc.le@tuni.fi).

A. S. M. Sayem is with the School of Engineering, Macquarie University, Sydney, NSW 2109, Australia (e-mail: abu-sadat-md.sayem@students.mq.edu.au). applied, a better resilience to machine washing was successfully demonstrated with some coatings. However, a major degradation in performance can still be seen as the number of washing cycle increases.

For instance, in the case of microstrip patch antenna encapsulated with thermoplastic polyurethane (TPU) in [7], [8], a drop in radiation efficiency of up to $5 \%$ was observed after six washing cycles. In our previous attempts on RFID tag antennas with acrylic, epoxy, moisture protection spray, textile glue, rubber latex glue, and polyvinyl acetate adhesive (PVA) encapsulations [4], [5], [9], [10], only with epoxy, moisture protection spray, and textile glue, the tags that were still operational after ten washing cycles can be achieved. However, in most of the cases, a decrease of more than $50 \%$ in the maximum read range of the tag was still observed after the $10^{\text {th }}$ cycle, together with a major shift (i.e., 5-8\%) in its resonance frequency. On the other hand, with other coatings, we often discovered that the tags became dysfunctional after the $3^{\text {rd }}$ cycle or even less [5], [10]. Moreover, some coatings such as acrylic, epoxy, and PVA become rigid after curing, thus compromising the antenna wearability [4], [9]. Another work using porous polyurethane web coating has been reported recently in [11]. A relatively stable gain after 15 washing cycles has been reported for a microstrip patch antenna encapsulated with such material. Nevertheless, similar to [7], [8], the effectiveness of the proposed approach for the antenna with lumped electronic component has not yet been demonstrated.

Our previous studies [4], [5], [9] revealed that, the primary challenges in achieving antenna washing durability involve the lack of physical robustness of the materials used, including those for coatings, to stand against the repetitive mechanical stress and liquid or chemical exposure during machine washing. It was also found challenging to achieve a robust integration between the conductive and non-conductive materials that can survive the harsh pressure of recurrent washings. Such problem also applies to the integration of lumped electronic component to a flexible material, which is required in some antenna cases. This is particularly crucial when non-galvanic contact solutions [12]-[14] are not an option, for instance considering antenna design simplicity.

Recently in [6], [15], we proposed a combination of two classes of materials, polydimethylsiloxane (PDMS) and conductive fabric, as a new method to realize robust flexible active and passive antennas. By taking advantage of PDMS percolation into pores of fabric, we achieved a good integration between the conductive and the non-conductive parts of the antenna, which is inherently weak. By applying PDMS as 
the antenna encapsulation, we maintained the integration of the lumped electronic tuning elements to the flexible fabric. As the result, with the proposed approach, we successfully demonstrated a flexible electronically tunable antennas that can survive extreme bending and machine washing. The latter, however, has been done with one washing cycle only.

In this paper, we expand further our study on PDMSconductive fabric composite, by exposing such materials combination to repetitive machine washings to validate further its durability. For this purpose, we fabricated several ultra-high frequency (UHF) radio frequency identification (RFID) passive tag antennas using this composite material and conducted wireless reliability investigations of the tags by testing their read range in free space after each washing cycle. A study to improve the interconnection of the antenna and lumped electronic component to survive the recurrent washings is also presented. The motivation of this whole study is to verify the applicability of the proposed method as a solution to the textile-antenna washability issue discussed above.

\section{TAg Antenna TOPOLOGY}

The topology of the antenna used for the purpose of this study is given in Fig. 1. The antenna simulations and optimizations were conducted using CST Microwave Studio 2018. The design comprises of a planar dipole with inductive loop matching that we have widely used in our previous studies [5], [9], [10], [16]. The radiator layer, which has a total length and width of $l_{t}$ and $w_{t}$, respectively, is realized with a highly conductive fabric, nickel-copper-silver coated nylon ripstop from Marktek Inc. The radiator is sandwiched between two PDMS layers, having a relative permittivity of 2.7 and a loss tangent of 0.01 according to our measurements with an Agilent 805070E Dielectric Probe Kit in the frequency of 800 to $1000 \mathrm{MHz}$. The simulations show that, the PDMS full encapsulation leads to $10.2 \%$ decrease in the antenna physical length. This can be understood due to an increase in the antenna electrical length after the addition of a dielectric material as PDMS, which shifts the antenna resonance to lower frequency. On the other hand, the antenna radiation efficiency drops from 90 to $71.4 \%$ due to the loss contributed by PDMS. Nevertheless, such topology allows for a better integration between the two classes of material, thanks to PDMS-PDMS bonding created through the fabric pores. At the same time, owing to the unique characteristics of PDMS, its combination with conductive fabric provides physical robustness from harsh wearable environments, which might not be achieved by the conductive fabric itself [3], [15]. The length and width of the PDMS layers are slightly larger than those of the conductive fabric to allow PDMS-PDMS sealing at the edge of the antenna, maximizing the antenna protection.

In simulations, the conductive part of the antenna was modeled as a box with the thickness of the conductive fabric, $0.13 \mathrm{~mm}$, as specified by the manufacturer, and was assigned an effective conductivity of $1.02 \times 10^{5} \mathrm{~S} / \mathrm{m}$. This value is basically an approximated effective conductivity of a composite material consisting of PDMS and the conductive fabric we use in this work, obtained from our previous study [15]. The NXP UCODE G2iL series RFID IC having a wake-up power

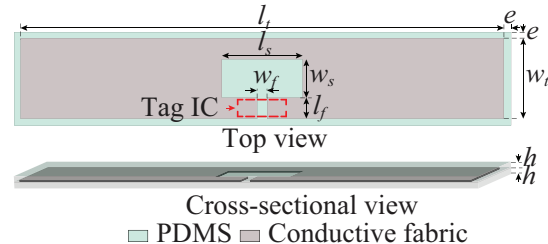

Fig. 1. Detailed geometry of the tag antenna $\left(l_{t}=96, w_{t}=16, l_{s}=16\right.$, $\left.w_{s}=7.3, l_{f}=3.6, w_{f}=2, e=2, h=0.5\right)$. All dimensions are in millimeters. of $-18 \mathrm{dBm}(15.8 \mu \mathrm{W})$ was chosen as the tag IC, which was modeled in the simulations as a parallel circuit of a $2.85 \mathrm{k} \Omega$ resistance and a $0.91 \mathrm{pF}$ capacitance.

In this study, the read range $\left(d_{\text {tag }}\right)$ in free space is used to quantify the performance of the tag antenna, estimated during the simulations through the function below [17]

$$
d_{\text {tag }}(\phi, \theta)=\frac{\lambda}{4 \pi} \sqrt{\frac{\chi_{p o l}(\phi, \theta) \tau e_{r} D(\phi, \theta) \mathrm{EIRP}}{P_{i c 0}}}
$$

where $\lambda$ is the wavelength of the reader's carrier signal, $\chi_{p o l}$ is the mutual polarization power efficiency between the tag and reader antennas, $e_{r}$ is the radiation efficiency of the tag antenna, $D$ is the directivity of the tag antenna, EIRP is the equivalent isotropic radiated power of the reader, $P_{i c 0}$ is the wake-up power of the tag IC, and $\tau$ is the power transfer efficiency of the antenna and IC. The latter can be determined through the relation between the impedances of the antenna $\left(Z_{\text {ant }}\right)$ and IC $\left(Z_{i c}\right)$, which is expressed as follows:

$$
\tau=\frac{4 \operatorname{Re}\left(Z_{a n t}\right) \operatorname{Re}\left(Z_{i c}\right)}{\left|Z_{a n t}+Z_{i c}\right|^{2}}
$$

The $l_{t}$ and $w_{t}$ of the tag were set to be 100 and $20 \mathrm{~mm}$ respectively, following the average dimensions of commercial passive tag antennas. On the other hand, some optimizations of $l_{s}$ and $w_{s}$ were conducted to achieve complex conjugate matching between the antenna and IC impedances at target frequency of $915 \mathrm{MHz}$. By doing so, a maximum power transfer between the two can be achieved, thus maximizing $d_{\text {tag }}$. This was done under the European RFID emission regulation $(\mathrm{EIRP}=3.28 \mathrm{~W}$ ) and with an assumption that the reader and tag antennas' polarizations are alligned $\left(\chi_{p o l}=1\right)$. The optimum dimensions of the antenna are given in the caption of Fig. 1.

\section{PROTOTYPE FABRICATION}

Layer-by-layer fabrication approach we described in [15] was applied for manufacturing the antenna prototypes. Each PDMS layer was prepared by pouring liquid PDMS into a customized ring-shaped mold having the required thickness, followed by curing in the oven. The conductive fabric was cut manually by means of a razor blade following the pattern in Fig. 1, and was adhered to the cured PDMS layer using uncured PDMS. The tag IC was connected to the antenna by attaching the pads of the IC fixture to the fabric using silver epoxy. To achieve good PDMS-PDMS layer bonding through the fabric, the PDMS solution for making the top layer was directly poured and cured over the cured bottom PDMS layer and conductive fabric.

It was revealed in our previous studies [9] that the attachment between the IC fixture and the textile antenna often fails 
after recurrent washings. Such occurrence seems to be caused by the fact that the area around the pad where the silver epoxy was applied, turns to be more rigid than the rest of the antenna including the coating. It is also worsened by the total size of the fixture that is comparable to the minimum bending curvature that can possibly happen to the antenna. Consequently, at certain point, this particular part cannot cope with the dynamics of the flexible antenna (e.g., the bending and twisting during washing or spinning), and thus are majorly impacted by the extreme mechanical deformation leading to detachment from the fabric.

To solve the above mentioned issue, we investigated three different configurations of the antenna-IC fixture interconnection. Figs. 2(a)-(c) show the three versions of the fabricated prototypes. Henceforth, they will be referred to as version $\mathrm{A}, \mathrm{B}$, and $\mathrm{C}$, respectively. Inspired by the mechanism behind the good integration of PDMS and conductive fabric [15], we opted to include some holes on the pads of the IC fixture as can be seen in version B and C (Figs. 2(b) and (c)). This is as opposed to the normal configuration in version A (Fig. 2(a)). Each circular hole on the pads of version B has a diameter of approximately $0.8 \mathrm{~mm}$, whereas the square hole on the pads of version $\mathrm{C}$ has a side of approximately $1.5 \mathrm{~mm}$. The holes were created by simply using needle and razor blade. The main aim of these holes is to facilitate the PDMS percolation, which results in PDMS-PDMS bonding through the pads. This bonding strengthens the attachment of the IC fixture to the fabric and simultaneously improves the flexibility of this area and thus reduces the impacts of deformation. For the purpose of verification of the findings, we fabricated three prototypes for each antenna version.

\section{WASHING DURABILITY EVALUATION}

All the antenna prototypes were washed repeatedly inside a household washing machine and their performance in terms of read range was tested after each washing cycle. Each washing was done with detergent and water $\left(40^{\circ} \mathrm{C}\right)$, in a complete cycle of cotton-cloth washing mode, having a total duration of 50 minutes and $900 \mathrm{rpm} / \mathrm{min}$ spinning speed. During washing, the antennas were put into a washing pouch and no other laundry was in the machine. Such procedure was repeated up to 15 times. The attainable read range of the tag antenna $\left(d_{t a g}\right)$ in free space was estimated from its measured threshold power, by using the relation shown below [16]

$$
d_{t a g}=\frac{\lambda}{4 \pi} \sqrt{\frac{\operatorname{EIRP} P_{t h *}}{\Lambda P_{t h}}}
$$

where $P_{t h}$ is the threshold power of the tag antenna obtained from the measurement using Voyantic Tagformance system in a polarization-matched configuration, while $\Lambda$ and $P_{t h^{*}}$ are the known sensitivity and the measured threshold power of the system reference tag, respectively. During the measurements, the same EIRP limit as used before in the simulations was applied.

The attainable maximum read range of all fabricated prototypes are given in Fig. 3. As can be seen, the whole prototypes achieve a very similar initial maximum read range, i.e., an average of $10.5 \mathrm{~m}$ with less than $2 \%$ relative standard deviation. This demonstrates the reproducibility of the

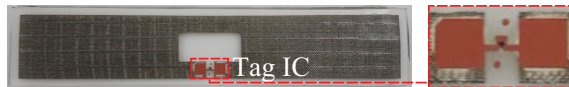

(a)

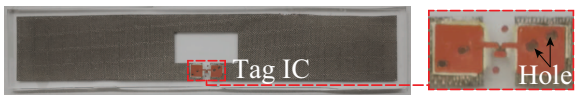

(b)

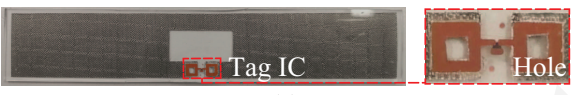

(c)

Fig. 2. Photographs of the fabricated tag antenna prototypes: (a) Version A. (b) Version B. (c) Version C. The difference among the three is on the pad modification of the tag IC as can be seen in the insets.

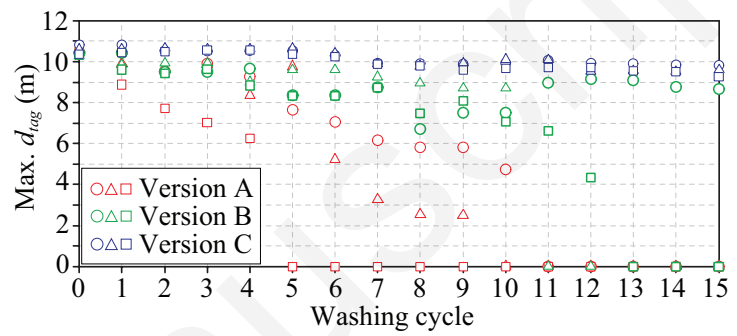

Fig. 3. Maximum read ranges of all fabricated prototypes after recurrent washings. The circle, triangle, and square symbols represent the first, second, and third prototypes of each antenna version.

proposed fabrication approach. At the same time, these results also suggest that the modifications on the antenna-IC fixture interconnection by adding holes, give a marginal effect to the initial performance of the tag. On the other hand, after washings, the three versions show very different performance one to another. From the trend of the results, a significant improvement in washing durability can be noted clearly from version $\mathrm{A}$ to $\mathrm{C}$. All version $\mathrm{C}$ prototypes remain functional after the $15^{\text {th }}$ washing cycle with a minimum degradation in the read range, whereas most of the version B prototypes fail after the $13^{\text {th }}$ washing cycle. On the other hand, all version $\mathrm{A}$ prototypes fail after the $11^{\text {th }}$ cycle and the degradation in their read range is found to be worst than versions $\mathrm{B}$ and $\mathrm{C}$.

For better observation, we have also plotted in Figs. 4(a)(c), the read range vs. frequency of one prototype from each version. In this case, we chose those which survived the most number of washing cycle. It is shown that, for version $\mathrm{A}$, the read range drops to $4.7 \mathrm{~m}$ after the $10^{\text {th }}$ washing cycle, which is approximately $54.8 \%$ lower than the initial range. It is also noticed that the peak shifts slightly to higher frequency. As the washing continues, the tags unfortunately become dysfunctional after the $11^{\text {th }}$ cycle, thus no range is indicated. On the other hand, prototypes version $\mathrm{B}$ and $\mathrm{C}$ remain operational even after the $15^{\text {th }}$ cycle. The results of version B suggest that the antenna robustness against washing is improved after the addition of two small circular holes on each pad of the IC fixture. This is indicated by the antenna read range, which only drops to $8.7 \mathrm{~m}$ (approximately $16.3 \%$ lower than the initial range) after the $15^{\text {th }}$ washing cycle. An even more superior performance is shown after the addition of bigger square holes on the fixture pads (see the results of version $\mathrm{C}$ ). The difference between the initial range and the read range after the $15^{\text {th }}$ cycle is found to be only $1 \mathrm{~m}$ lower 


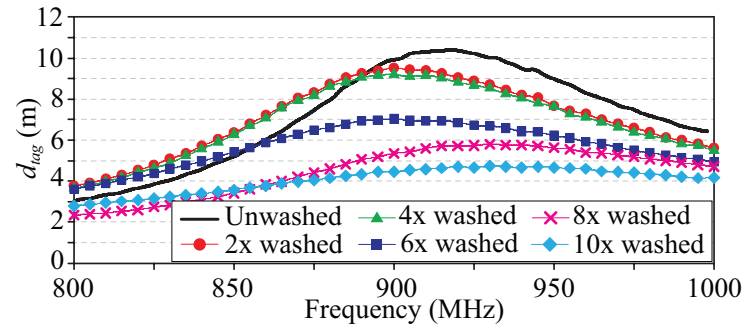

(a)

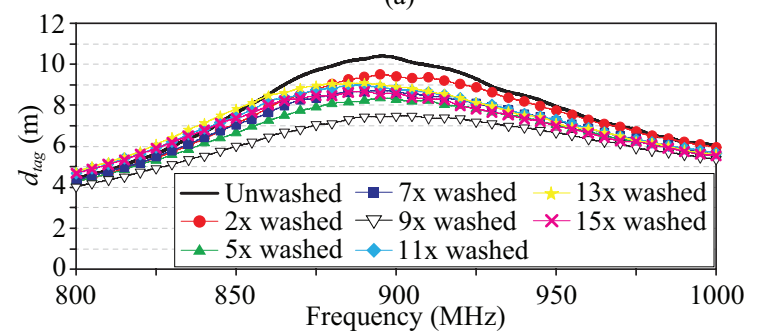

(b)

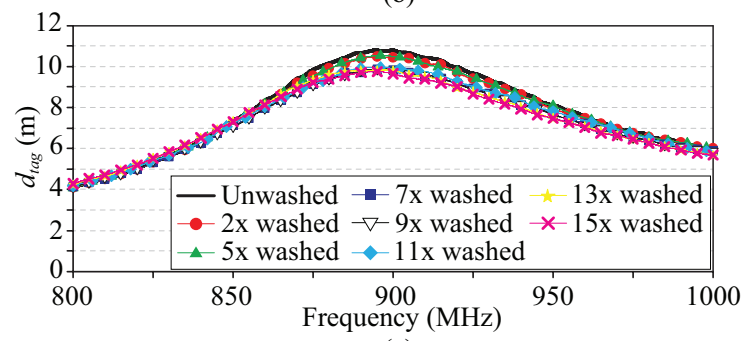

(c)

Fig. 4. Measured read ranges of the tag antennas after recurrent washings: (a) Version A. (b) Version B. (c) Version C.

or approximately $9.3 \%$ and such performance appears to be maintained quite stably since the $7^{\text {th }}$ washing cycle.

The fact that the three prototype versions were fabricated following the same topology and procedure, except for the interconnection of the IC fixture and the antenna, it can therefore be concluded as follows. The proposed materials combination, PDMS-embedded conductive fabric, indeed possesses the physical robustness required to stand against liquid or chemical exposure and mechanical pressure of repetitive machine washings. It is clear from the results in Figs. 3 and 4 that, the issue of degradation in the antenna read range after washing, does not come from the conductive fabric or the coating layer but majorly from the antenna-IC fixture interconnection. In fact, we observed that on the body of the properly fabricated prototypes, there were no visual traces of fractures or cracks, neither on the PDMS layers nor on the fabric, even after the $15^{\text {th }}$ cycle of washings. The shape of the three prototype versions were also retained without breaking, thanks to the superior flexibility of this composite material. These observations suggest that, by utilizing this composite material, the dissolution of the fabric's conductive materials can be prevented and a well-preserved electrical connectivity can be expected after repetitive machine washings. There were indeed found some minor delaminations of the PDMS from the conductive fabric, which is the consequence of low porosity level of the fabric used, as shown previously in [15]. Such occurrence is shown in Fig. 5, which was also found in version A and B (not shown here). However, most importantly, the conductive fabric remained sealed inside the

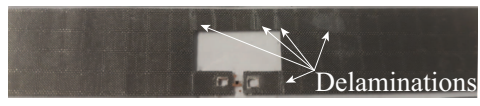

Fig. 5. Back view photograph of one of the prototypes version $\mathrm{C}$ after the $15^{\text {th }}$ washing cycle. The pointed marks indicate the area with delaminations.

PDMS coatings, thus was still protected. The use of other type of conductive fabric with higher porosity can be done to avoid such issue, however, with a consequence of having lower effective conductivity of the antenna radiator [15] and hence the read range.

As implied by the results in Figs. 3 and 4, a further treatment is nevertheless required when PDMS-conductive fabric composite is applied for the realization of a textile antenna incorporating lumped electronic component. Although the PDMS coating has been successful in protecting the lumped component from some possible risks (e.g., exposure to liquid or washing chemicals, being washed away or peeled off while being worn or machined-washed), the integration of the lumped component to the body of such flexible antenna has to be improved, to survive harsh pressures of repetitive machine washings. The treatment might be different from one case to another, depending on the size and configuration of the lumped component. For the case of UHF RFID tag antenna we used in this work, we incorporated holes on the pads of the IC fixture. The results in Figs. 3 and 4 validate the effectiveness of the PDMS-PDMS bonding formed through the holes on the pads in strengthening the attachment of the IC tag fixture to the fabric antenna. The more spaces created for the PDMS percolation, which allows for the formation of PDMSPDMS bonding through the pads, the more robust the antennaIC interconnection becomes. This is validated by the three version $\mathrm{C}$ prototypes, which consistently show a more stable performance as compared to the versions A and B (Fig. 3).

\section{CONClusion}

We have successfully demonstrated the applicability of PDMS-conductive fabric composite for realization of flexible wearable antennas that can withstand multiple washing cycles. Through the absence of fractures or cracks, liquid exposure in the fabric, and other permanent physical deformations on the body of the fabricated tag antennas after 15 washing cycles, it is demonstrated that PDMS-embedded conductive fabric composite has the physical robustness required to survive the harsh environment of recurrent machine washings. However, for the case of flexible antenna incorporating lumped electronic component, modification during the fabrication, particularly on the interconnection between the antenna and the lumped component might be required. This has been validated through the results of the tag antennas with square holes incorporated on the pads of the fixture. Unlike previously reported results, such modification allows the antenna to maintain a minimum degradation in performance after multiple washings, i.e., only 9.8\% drop in maximum read range with stable resonance frequency after 15 washing cycles, which has been maintained stably since the $7^{\text {th }}$ cycle. As future work, more washing cycles with longer duration are considered to be conducted to investigate further the durability of the proposed method. 


\section{REFERENCES}

[1] R. Salvado, C. Loss, R. Gonçalves, and P. Pinho, "Textile materials for the design of wearable antennas: a survey," Sensors, vol. 12, no. 11, pp. $15841-15857,2012$.

[2] M. Stoppa and A. Chiolerio, "Wearable electronics and smart textiles: A critical review," Sensors, vol. 14, no. 7, pp. 11957-11 992, 2014.

[3] B. Mohamadzade, R. M. Hashmi, R. B. V. B. Simorangkir, R. Gharaei, S. U. Rehman, and Q. H. Abbasi, "Recent advances in fabrication methods for flexible antennas in wearable devices: State of the art," Sensors, vol. 19, no. 10, p. 2312, 2019.

[4] T. Kellomäki, J. Virkki, S. Merilampi, and L. Ukkonen, "Towards washable wearable antennas: A comparison of coating materials for screen printed textile based UHF RFID tags," Int. Journal of Ant. and Propag., vol. 2012, no. 476570, pp. 1-11, 2012.

[5] Y. Y. Fu, Y. L. Chan, M. H. Yang, Y. C. Chan, J. Virkki, T. Bjorninen, L. Sydanheimo, and L. Ukkonen, "Experimental study on the washing durability of electro-textile UHF RFID tags," IEEE Antennas Wireless Propag. Lett., vol. 14, pp. 466-469, 2015.

[6] R. B. V. B. Simorangkir, Y. Yang, K. P. Esselle, and B. A. Zeb, "A method to realize robust flexible electronically tunable antennas using polymer-embedded conductive fabric," IEEE Trans. Antennas Propag., vol. 66, no. 1, pp. 50-58, Jan 2018.

[7] M. L. Scarpello, I. Kazani, C. Hertleer, H. Rogier, and D. V. Ginste, "Stability and efficiency of screen-printed wearable and washable antennas," IEEE Antennas Wireless Propag. Lett., vol. 11, pp. 838-841, 2012.

[8] I. Kazani, M. L. Scarpello, C. Hertleer, H. Rogier, G. de Mey, G. Guxho, and L. V. Langenhove, "Washable screen printed textile antennas," Adv. in Science and Tech., vol. 80, pp. 118-122, 2013.

[9] S. Wang, N. L. Chong, J. Virkki, T. Björninen, L. Sydänheimo, and L. Ukkonen, "Towards washable electrotextile UHF RFID tags: reliability study of epoxy-coated copper fabric antennas," Int. Journal of Ant. and Propag., vol. 2015, no. 424150, pp. 1-8, 2015.

[10] M. Guibert, A. Massicart, X. Chen, H. He, J. Torres, L. Ukkonen, and J. Virkki, "Washing reliability of painted, embroidered, and electrotextile wearable RFID tags," in Proc. Prog. in Electromagnetics Res. Symposium (PIERS), 2017, pp. 828-831.

[11] H. Shahariar, H. Soewardiman, C. A. Muchler, J. J. Adams, and J. S. Jur, "Porous textile antenna designs for improved wearability," Smart Materials and Structures, vol. 27, p. 045008, 2018.

[12] F. Alimenti, M. Virili, G. Orecchini, P. Mezzanotte, V. Palazzari, M. M. Tentzeris, and L. Roselli, "A new contactless assembly method for paper substrate antennas and UHF RFID chips," IEEE Trans. Microw. Theory Techn., vol. 59, no. 3, pp. 627-637, 2011.

[13] M. Virili, H. Rogier, F. Alimenti, P. Mezzanotte, and L. Roselli, "Wearable textile antenna magnetically coupled to flexible active electronic circuits," IEEE Antennas Wireless Propag. Lett., vol. 13, pp. 209-212, 2014.

[14] T. Menezes, F. R. da Silva, F. L. Cabrera, and F. R. de Sousa, "Design and measurement of a contactless interface between a dipole antenna and a CMOS fully integrated wireless power receiver," IET Microw. Ant. and Propag., vol. 12, no. 8, pp. 1255-1259, 2018.

[15] R. B. V. B. Simorangkir, Y. Yang, R. M. Hashmi, T. Bjorninen, K. P. Esselle, and L. Ukkonen, "Polydimethylsiloxane-embedded conductive fabric: characterization and application for realization of robust passive and active flexible wearable antennas," IEEE Access, vol. 6, pp. 48102 $48112,2018$.

[16] J. Virkki, T. Bjorninen, S. Merilampi, L. Sydanheimo, and L. Ukkonen, "The effects of recurrent stretching on the performance of electro-textile and screen-printed ultra-high-frequency radio-frequency identifications tags," Textile Res. Journal, vol. 85, no. 3, pp. 294-301, 2015.

[17] G. Marrocco, "The art of UHF RFID antenna design: Impedancematching and size-reduction techniques," IEEE Antennas Propag. Mag., vol. 59, no. 1, pp. 66-79, 2008. 